\title{
Dynamics of transformation of ultrasonic vibrations in twisted waveguides
}

\author{
Sergey B. Kudryshev*, Valentin S. Minakov, Alexey A. Zakaluyzhnyy, \\ Vladimir A. Peglivanyan \\ Don State Technical University, 344000 Rostov-on-Don Gagarin sq. 1, Russia
}

\begin{abstract}
The effective use of ultrasound energy in various technological processes largely depends on the type of ultrasonic vibrations. Wide application in practice has found longitudinal ultrasonic fluctuations in connection with simplicity of realization, and also presence of the developed theoretical and settlement base. The longitudinal-torsional ultrasonic oscillations, the realization of which practically does not differ from the realization of longitudinal oscillations, and the efficiency and technological flexibility are much higher than for longitudinal or torsional ultrasonic oscillations, practically no applications have been found. This is due to several circumstances and, above all, from the theoretical basis for the transformation of this type of oscillation, since the theory of compressedtwisted rods was developed mainly in connection with the problem of longitudinal stability. The article deals with the case of transformation of longitudinal ultrasonic oscillations into complex ones by attaching to the last stage of the concentrator of the longitudinal acoustic system of the twisted waveguide.
\end{abstract}

\section{Introduction}

The wide and effective use of ultrasound in different processes largely depends on the type of oscillation. Application of ultrasonic vibrations in various processes found the use of longitudinal, torsional and integrated fluctuations. In connection with the ease of implementation, well-designed mathematical models and calculation bases, wide use in practice found longitudinal ultrasonic vibrations. Torsional ultrasonic vibrations have not found wide application due to the complex methods of implementation. Complex fluctuations include the coupling of longitudinal and torsional vibrations. The implementation of such fluctuations does not differ from longitudinal, and their effectiveness and technological flexibility is much higher.

The expansion of effective use of energy in the technological practice of complex narrow can be associated with the development of the theory of transformation of this type of oscillations. The theory will allow to consider the dynamics of complex oscillations in a form convenient for engineering calculation, and, in addition to the basic formulas, to give a General method of calculation.

\footnotetext{
*Corresponding author: skudryshov@donstu.ru
} 
The formation of complex oscillations is possible through the use of a naturally twisted waveguide connected to the last stage of the concentrator of the longitudinal acoustic system. The consideration of the twisted waveguide as a naturally twisted rod $[1,2,3]$, subject to the action of forces arising in the process or other type of processing leads to a certain dynamic three-dimensional problem.

\section{Mathematical model. Equation of motion}

When developing the dynamics of a twisted waveguide, a mathematical model was obtained. It allowed to simplify the research and showed its behavior in any cross section quite accurately $[5,6,7,9]$.

The averaged movement of the cross-section as a whole is considered in the construction of a mathematical model. Although in the process of operation the cross-section of the waveguide does not remain flat and experiences a complex deformation $[4,8]$.

In addition, the mathematical model of the waveguide was based on the following facts obtained from the experiment and the solution of the corresponding static problems [1]:

- tension - compression of the waveguide by longitudinal force in the absence of external torque is accompanied by its torsion deformation;

- twisting of the waveguide by external torque in the absence of longitudinal force is accompanied by its lengthening or shortening;

the magnitude and direction accompanying the deformation of the waveguide (in the absence of torsion torque and the tensile (compression) in the absence of the tensile (compressive) force) is determined by the material of the waveguide and its geometric characteristics (length of the twisted portion, the twist angle, cross sectional area etc.).

Consider in figure 1 waveguide, in a fixed coordinate system $\mathrm{X}, \mathrm{Y}, \mathrm{Z}$, choosing the plane of one of the normal sections of the unloaded waveguide for the coordinate plane (Y, Z) and directing the $\mathrm{x}$ axis along the axis of the waveguide.

We introduce two values in the mathematical model: the longitudinal movement $U$ of the cross-section $\mathrm{X}$ and $\varphi$ - the angle of its rotation relative to the natural (undeformed) state.

Consider the waveguide section lying to the right of section $X$ (Fig. 1), in order to maintain the previous state, the force $T(x, t)$ directed along the ox axis and the torque relative to this axis equal to $M(x, t)$ must be applied to the section $\mathrm{X}$.

To obtain the differential equations of motion of the waveguide let us consider the time $\mathrm{t}-$ land, limited to two infinitely close cross-sections $x$ and $x+\Delta x$. Using this plot, the main theorem the dynamics of the system about the amount of motion and kinematic moment about the axis $\mathrm{OX}$, considering the given above assumptions, we find that when $\Delta \mathrm{x} \rightarrow 0$ :

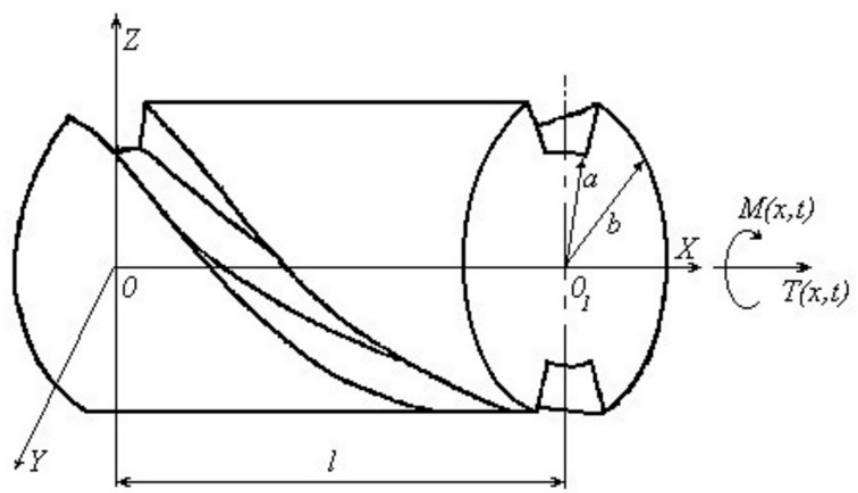

Fig. 1. The scheme of the forces applied to the cross section $X$. 


$$
\rho \cdot \Omega \frac{\partial^{2} U}{\partial t^{2}}=\frac{\partial T}{\partial x}, \rho \cdot J_{\Omega} \cdot \frac{\partial^{2} \phi}{\partial t^{2}}=\frac{\partial M}{\partial x} .
$$

where: $\rho$-density of the waveguide material; $\Omega$ - cross-sectional area of the waveguide; $J_{\Omega}$ - moment of inertia of the cross-sectional area relative to its center.

Equation (1) becomes more specific, given the elastic nature of the deformation of the waveguide.

$$
\begin{gathered}
T=\alpha_{11} \cdot \varepsilon+\alpha_{12} \cdot \theta, M=\alpha_{21} \cdot \varepsilon+\alpha_{22} \cdot \theta \\
\alpha_{11}=E \cdot \Omega ; \alpha_{12}=\alpha_{21}=\tau_{0} \cdot E \cdot\left(J_{\Omega}-T_{0}\right) ; \alpha_{22}=G \cdot T .
\end{gathered}
$$

where: $E$ - modulus of elasticity; $\tau_{0}$ - relative angle of rotation of the waveguide; $T_{0}=\int_{\Omega}\left(R^{2}+\dot{\phi} \dot{\prime}\right) d \Omega ;-$ geometric torsional rigidity of the untwisted waveguide; $G$ - shear module;

$$
T=T_{0}+2 \cdot(1+v) \cdot \theta_{0}^{2} \cdot\left(J_{r}^{0}-T_{r}^{0}\right) .
$$

where $\quad v-$ Poisson's ratio, $\quad J_{r}^{0}=\int_{\Omega} R^{4} d \Omega ; T_{r}^{0}=\int_{\Omega}\left(R^{4}-\left(\phi_{\psi}^{\prime}\right)^{2}\right) d \Omega ; \quad \phi_{\psi}^{\prime}=y \frac{\partial \phi}{\partial z}-z \frac{\partial \phi}{\partial y}$. $\varphi$ - torsion function of the corresponding unbroken waveguide.

Expressing the deformation components $\varepsilon$ and $\theta$ through the displacement $U$ and the angle $\varphi$ by the known Cauchy formulas [2]

$$
\varepsilon=\frac{\partial U}{\partial x} ; \quad \theta=\frac{\partial \phi}{\partial x}
$$

after, using equality (2), we transform equation (1) to form:

$$
\begin{gathered}
\left\{\begin{array}{l}
\frac{\partial^{2} U}{\partial t^{2}}=\beta_{11} \frac{\partial^{2} U}{\partial x^{2}}+\beta_{12} \frac{\partial^{2} \phi}{\partial x^{2}} \\
i_{0}^{2} \frac{\partial^{2} \phi}{\partial t^{2}}=\beta_{21} \frac{\partial^{2} U}{\partial x^{2}}+\beta_{22} \frac{\partial^{2} \phi}{\partial x^{2}}
\end{array}\right. \\
i_{0}^{2}=\frac{J_{\Omega}}{\Omega} ; \quad \beta_{k j}=\frac{\alpha_{k j}}{\rho \cdot \Omega},(k=1,2),(j=1,2) .
\end{gathered}
$$

\section{Oscillations of the unloaded waveguide}

The work was carried out within the framework of initiative research

When studying the steady-state oscillations of an unloaded waveguide, in which the extreme cross-section of the swirled part makes longitudinal harmonic oscillations with a constant frequency and amplitude, the following boundary conditions for $U(x, t)$ and $\varphi(x, t)$ were established [7].

$$
\begin{aligned}
& U=A \cdot e^{i \cdot k \cdot t}, \quad \phi=0 \text { if } x=0 ; \\
& T=M=0, \quad \text { if } x=l .
\end{aligned}
$$

We will look for a solution in the form:

$$
V_{m}(x, t)=A_{m}(x) \cdot e^{i \cdot k \cdot t} .
$$


After some transformations [7] we obtain:

$$
U(x, t)=U_{0}(x) \cdot e^{i \cdot k \cdot t}, \quad \phi(x, t)=\phi_{0}(x) \cdot e^{i \cdot k \cdot t},
$$

where $U_{0}(x)$ and $\varphi_{0}(x)$ are the amplitude factors determined by the formulas:

$$
\begin{aligned}
& U_{0}(x)=\frac{\alpha_{21} \cdot A}{R o} \cdot \frac{a_{1} \cos \left(\frac{k}{c_{1}}(l-x)\right) \cdot \cos \frac{k \cdot l}{c_{2}}-a_{2} \cos \left(\frac{k}{c_{2}}(l-x)\right) \cdot \cos \frac{k \cdot l}{c_{1}}}{\cos \frac{k \cdot l}{c_{1}} \cdot \cos \frac{k \cdot l}{c_{2}}}, \\
& \varphi_{0}(x)=\frac{\alpha_{21} \cdot A}{R_{o}} \cdot \frac{\cos \left(\frac{k}{c_{1}}(l-x)\right) \cdot \cos \frac{k \cdot l}{c_{2}}-\cos \left(\frac{k}{c_{2}}(l-x)\right) \cdot \cos \frac{k \cdot l}{c_{1}}}{\cos \frac{k \cdot l}{c_{1}} \cdot \cos \frac{k \cdot l}{c_{2}}} .
\end{aligned}
$$

Analyzing the ratio of the oscillation amplitude of the waveguide end at $\mathrm{x}=1$

$$
\frac{U_{0}(l)}{\phi_{0}(l)}=\frac{a_{1} \cdot \cos \frac{k \cdot l}{c_{2}}-a_{2} \cdot \cos \frac{k \cdot l}{c_{1}}}{\cos \frac{k \cdot l}{c_{2}}-\cos \frac{k \cdot l}{c_{1}}}
$$

We can conclude that the resonance, when $\cos \frac{k \cdot l}{c_{m}}=0$ :

$$
\frac{U_{0}(l)}{\phi_{0}(l)}=a_{m},(m=1,2)
$$

Having received a mathematical model of the dynamics of the longitudinal-torsional ultrasonic waveguide, it became possible to construct the AFC for this type of waveguides. For analysis were selected waveguides of titanium alloy VT5 and Steel 45. The calculated (dashed line) and experimental (solid line) frequency response are shown in figures 2 and 3.

Analysis presented in Fig. 2 and 3 the frequency response for a complex waveform shows their good agreement. This fact can be the basis for considering that the obtained mathematical model, the assumptions made, as well as the boundary conditions set are correct.

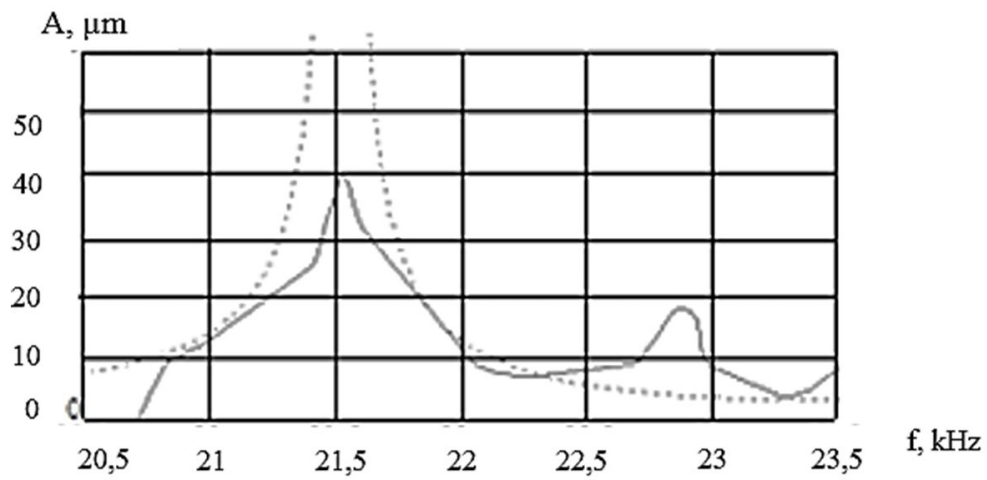

Fig. 2. Frequency response of longitudinal-torsional waveguide for VT5. 


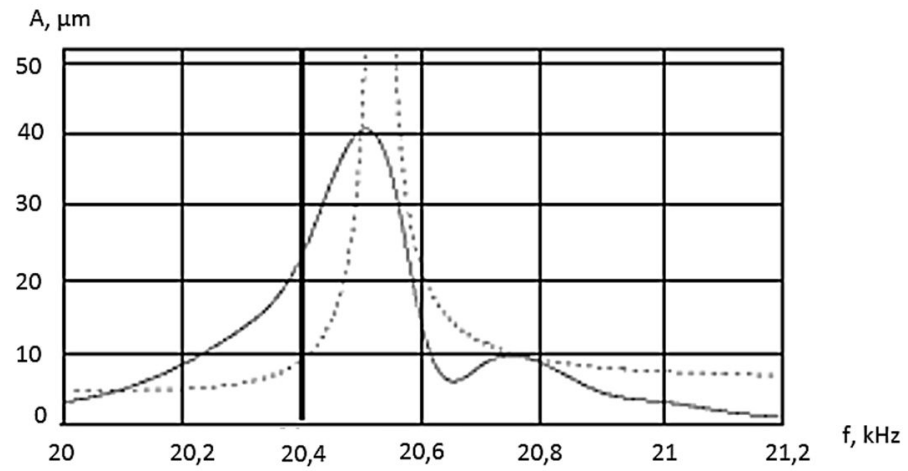

Fig. 3. The frequency response of a longitudinal-torsional waveguide for the Steel 45.

\section{Conclusion}

The analysis of the obtained ratio (12) and the sign of the coefficient $a_{m}$ in (13) makes it possible to assume that the difference between the two resonance frequencies $f_{1}$ and $f_{2}$ is that at a frequency close to one resonance frequency, the longitudinal and torsional components will be in phase, and to the other - is in the antiphase. This conclusion allows us to obtain two limiting states, when only one component is present in the complex oscillations: longitudinal or torsional. This property can be widely used in practice to control the type of vibrations when using complex ultrasonic vibrations.

Having considered the dependence of the amplitude of complex ultrasonic vibrations (figure 2 and 3 ) on the obtained mathematical model and comparing them with the experiments, we can draw the following conclusions:

1. Changing the geometry and material for the waveguide leads to a change in its frequency response.

2. By reducing the angle recess of the waveguide in the plane of the resonant peaks to the main resonance frequency and sub-harmonics, which become closer, with the frequency of the fundamental resonance is reduced and the sub-harmonics, which increases.

3 . The increase in the ratio of the outer radius and the radius of the recess of the naturally twisted waveguide leads to a decrease in the resonance frequency. The difference between the main resonance frequency and the subharmonic frequency does not change significantly.

4. The quality of the waveguide material affects the frequency of the main resonance and subharmonic. So, when the quality of the material decreases, the amplitude-frequency characteristic is shifted to the lower frequency range, and the frequency range between the main resonance and the subharmonic increases.

\section{References}

1. A.K. Rukhadze, Applied Mathematics and Mechanics. On deformation of naturally twisted rods, 11, 5, 533 (1947)

2. P.M. Reese, Deformation of naturally twisted rods, DAN, USSR, 23, 1, 18-21, 5, 441 (1939)

3. I.A. Birger, Strength, stability, oscillation, Moscow, Mechanical Engineering, 1, 253 (1968) 
4. B.F. Shore, Towards a theory of swirling nonuniformly heated rods. Proceedings of the USSR Academy of Sciences, OTN, Mechanics and Mechanical Engineering, 1, 136 (1960)

5. V.S. Minakov, Dynamics of Oscillating Drills, Abstracts of the 7th All-Union Acoustic Conference, Leningrad, 212 (1971)

6. V.S. Korchagin, On the dynamics of naturally twisted waveguide rods, Rostov-on-Don, RISKhM, 11 (1983)

7. V.S. Minakov, To the problem of longitudinal-torsional dynamics, Izvestiya SKNTSVSh, Technical Sciences, 6, 71 (1987)

8. N.H. Harutyunyan, B.L. Abrahamyan, Torsion of elastic bodies (Moscow, Fizmatgiz, 1963)

9. S.B. Kudryashev, Development of the dynamics of longitudinal-torsion waveguides with respect to the process of electro-acoustic spraying with hardening of cutting tools: author's abstract. phd, (Rostov-on-Don, 1998) 